\title{
Pinning Improvement of A15 Applied Superconducting Materials
}

\author{
B.A. GLOWACKI ${ }^{a, b, c, *}$ \\ ${ }^{a}$ Department of Materials Science and Metallurgy, University of Cambridge, \\ 27 Charles Babbage Road, Cambridge CB3 0FS, England \\ ${ }^{b}$ Department of Physics and Energy, Bernal Institute, University of Limerick, Castletroy, Ireland \\ ${ }^{c}$ Institute of Power Engineering, Mory 8, 01-330 Warsaw, Poland
}

\begin{abstract}
Electron-phonon coupling is one of the most common interaction in superconducting materials ranging from $\mathrm{Nb}_{3} \mathrm{Sn}, \mathrm{MgB}_{2}$, iron-based superconductors and high temperature cuprates such as $\mathrm{YBa}_{2} \mathrm{Cu}_{3} \mathrm{O}_{7-\delta}$ and $\mathrm{HgBa}_{2} \mathrm{Ca}_{2} \mathrm{Cu}_{3} \mathrm{O}_{8+\delta}$. However an importance of the electron-phonon coupling constant, $\lambda_{e p}$, should not be underestimated for characterisation of the electronic properties of superconducting materials, but it is important that the enhanced flux pinning mechanism can be hold responsible for the applicability of the A15 superconducting materials in emerging hydrogen cryomagnetic technology where temperature of the liquid $\mathrm{H}_{2}$ can be as low as $14 \mathrm{~K}$.
\end{abstract}

DOI: 10.12693/APhysPolA.130.531

PACS/topics: 74.25.Wx, 74.25.Sv, 74.25.Op

\section{Introduction}

The discovery of high temperature superconducting materials, HTS, and medium temperature superconducting material, MTS, $\left(\mathrm{MgB}_{2}\right)$, in 1986 and 2001 correspondingly does not eliminate the old well-established Nb-based conductors, but in contrary lack of the reliable strong, long lengths, high current at high magnetic filed HTS and MTS conductors brought a renaissance to the Nb-based technologies for large-scale applications in 21th century. The well-established Nb-based low temperature superconductors, LTS, such as $\mathrm{NbTi}$ and $\mathrm{Nb}_{3}$ Sn represent $98 \%$ world production of superconducting materials, where the remaining $2 \%$ is shared between $\mathrm{Nb}_{3} \mathrm{Al}, \mathrm{MgB}_{2}$ as well as high temperature superconductors (HTS) such as $\mathrm{Bi}$-based, $\mathrm{REBa}_{2} \mathrm{Cu}_{3} \mathrm{O}_{7-x}$. Despite the predicted raising prices of the liquid helium [1] but taking into consideration rapid progress in cryogenic cooling technologies there is an urgent need to improve even further the high magnetic field performance of the LTS conductors for applications.

There is an important question to be answered if the fundamental pinning properties of (A15) $\mathrm{Nb}_{3} \mathrm{Sn}$ and $\mathrm{Nb}_{3} \mathrm{Al}$ and $\mathrm{Nb}_{3} \mathrm{Ga}$ conductors can be improved by changing the pinning mechanism to continue to compete with emerging MTS and HTS materials.

Recent intensive research on powder-in-tube $\mathrm{NbSn}_{2}{ }^{-}$ $\mathrm{Cu}-\mathrm{Sn}$ composites results in exceptionally high $J_{c}$ of a very fine $\mathrm{Nb}_{3} \mathrm{Sn}$ layer formed [2]. However a substantial improvement of $J_{c}(B)$ is well documented but there is no change in a pinning mechanism of the $\mathrm{Nb}_{3} \mathrm{Sn}$ layers which will move the maximum pinning force towards the higher magnetic flux density normalised values $B / B_{c 2}$.

\footnotetext{
*corresponding author; e-mail: bag10@cam.ac.uk
}

The performance of the HTS materials very much depends on the magnetic flux density to which the material is exposed to, therefore optimization of the pinning mechanism for the applications in the case of HTS is even more complex than it is in the case of LTS and as such will not be discussed in the current manuscript. Pinning in $\mathrm{MgB}_{2}$ in situ and ex situ powder in tube conductors appears to be possible to modify assisted by the mechanical deformation and compression stress, however difference between magnetic flux pinning performance for powder in tube and coated conductors is dramatically different and direction for the improvement has well defined target [3].

In the current manuscript we will concentrate on $\mathrm{Nb}_{3} \mathrm{Sn}$ conductors where by altering the typical grain boundary structure originating from a simple equiaxed grains to elongated ribbon-like grain-reinforced microstructure could be the most plausible solution for high field pinning improvement. Further improvement of intragranular pinning in rapidly quenched $\mathrm{Nb}_{3}(\mathrm{Al}, \mathrm{Ge})$ and $\mathrm{Nb}_{3} \mathrm{Ga}$ conductors by carefully optimised thermomechanical treatment looks also promising.

\section{Critical parameters and global pinning force of A15 Nb-based conductors}

The most important dependences of superconducting parameters characterising the applicability of the particular materials is $B_{c 2}(T)$ in Fig. 1 and obviously pinning force, $F_{p}$, see Eq. (1) and Table I.

In most high pinning type II superconductors the global pinning force frequently exhibits scaling behaviour with reduced flux density $b=B / B_{c 2}$, The significance of this equation is that if one measures $F_{p}$ vs. $b$ at one temperature, then $F_{p}$ at other temperatures can be predicted by scaling the results with a factor $\left[B_{c 2}(T)\right]^{n}$ : 


\section{TABLE I}

Superconducting properties of some A15 materials; $B_{c 2}$ given at $4 \mathrm{~K}$.

\begin{tabular}{c|c|c}
\hline \hline Compound & $T_{c}[\mathrm{~K}]$ & $B_{c 2}[\mathrm{~T}]$ \\
\hline $\mathrm{Nb}_{3} \mathrm{Sn}$ & 18.5 & 24 \\
$\mathrm{Nb}_{3} \mathrm{Al}$ & 18.9 & 29.5 \\
$\mathrm{Nb}_{3} \mathrm{Ge}$ & 23.2 & 38 \\
$\mathrm{Nb}_{3}(\mathrm{Al}, \mathrm{Ge})$ & 21.0 & 44
\end{tabular}
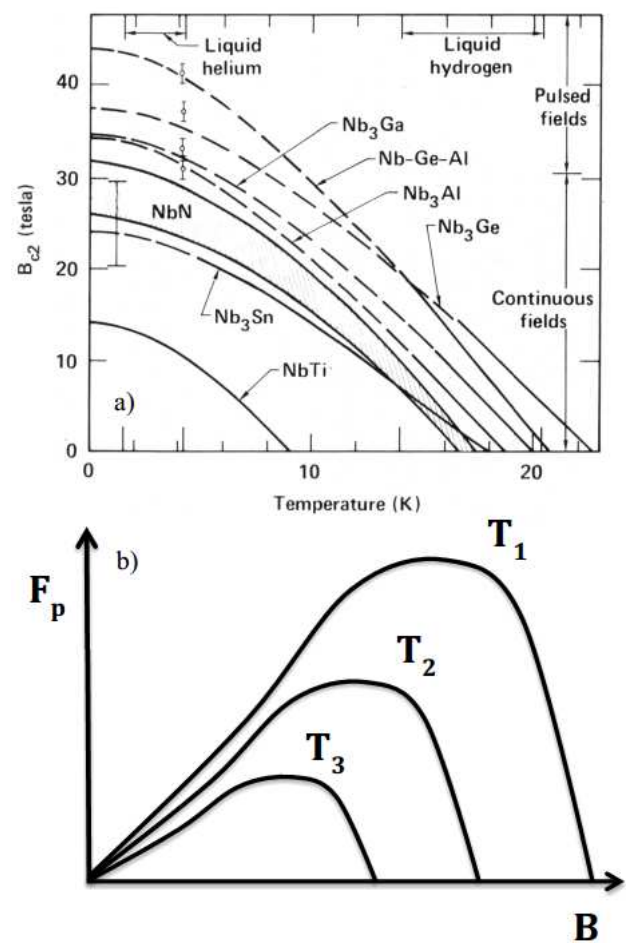

Fig. 1. (a) The upper critical flux density versus temperature of Nb-based conductors after [4]; one can notice that melting temperature of the hydrogen which is so-called slash hydrogen used in the spacecraft has a temperature of the $14 \mathrm{~K}$ at which the applicability of the $\mathrm{Nb}_{3}(\mathrm{Ge}, \mathrm{Al})$ can be considered at the range of $15 \mathrm{~T}$ [5]. (b) Volume pinning force versus temperatures for exemplary A15 superconductor showing that established maximum at high normalised magnetic flux density is beneficial for performance at higher temperatures $F_{p}(T)=\left(J_{c} B\right)_{T}$, where temperatures $T_{3}>T_{2}>T_{1}$.

$$
\begin{aligned}
& F_{p}=J_{c} B=G \frac{\left(B_{c 2}(T)\right)^{2}}{k^{m}} f(b), \\
& f(b)=b^{p}\left(1-b^{q}\right),
\end{aligned}
$$

where constant $G$ is a geometrical factor of the microstructure (usually interpreted as a surface area of inclusion per unit volume of matrix), $n$ and $m$ are fitting parameters, $k$ is smoothing parameter defining maximum pinning value, and $p$ and $q$ are pinning and materials related parameters. The position of the maximum of the pinning force on the coordinate axis of reduced flux density $b$ is defined by the ratio $p /(p+q)$. Kramer [6] investigated the experimental data of several groups of
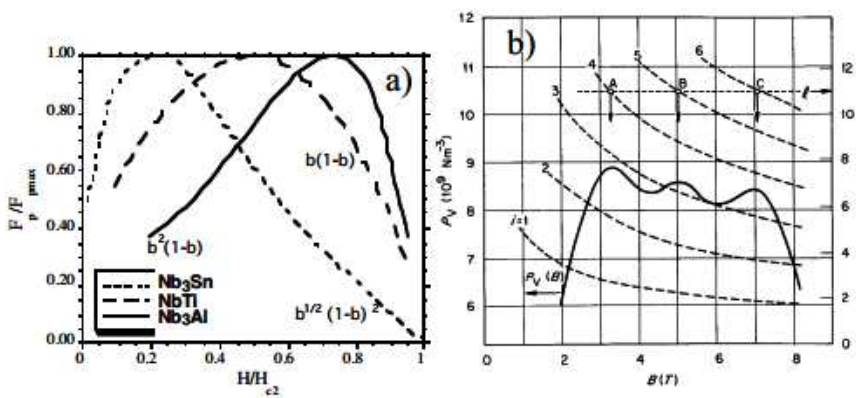

Fig. 2. (a) Normalised pinning force versus normalised magnetic field dependences for Nb-based conductors: $\mathrm{NbTi}, \mathrm{Nb}_{3} \mathrm{Sn}$, and $\mathrm{Nb}_{3} \mathrm{Al}$ (rapidly quenched). (b) Volume pinning force density $P_{v}$ as a function of $B$ in NbTi conductor containing an ordered array of $\alpha$-Ti particles, after Ullmaier [7].

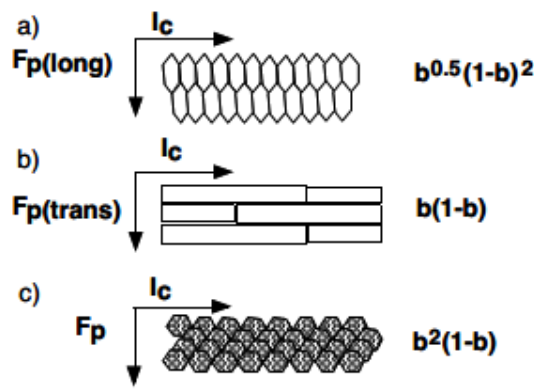

Fig. 3. Fragments of the cross-sectional grain structure of the superconducting filament along the wire and current direction: (a) equiaxed grain structure characteristic for $\mathrm{Nb}_{3} \mathrm{Sn}$ conductors, (b) elongated grain structure typical for NbTi conductors, (c) fine defect substructure typical for rapidly quenched $\mathrm{Nb}_{3} \mathrm{Al}$ and $\mathrm{Nb}_{3} \mathrm{Ga}$.

hard superconductors such as NbZr, NbTa, NbTi, NbHf, $\mathrm{Nb}_{3} \mathrm{Sn}, \mathrm{Nb}_{3} \mathrm{Al}$, and $\mathrm{V}_{3} \mathrm{Ga}$, and pointed out that Eq. (1) can indeed describe the pinning force with the power $n$ varying between 2 and 3 , and with the function $f(b)$ from Eq. (2) depending on the microstructure and metallurgical treatment.

There are three main categories of Nb-based superconducting materials, which are described by the normalised pinning force dependences versus normalised magnetic field, $f(b)$ (Eq. (2)). Most of the $\mathrm{Nb}_{3} \mathrm{Sn}$ conductors are close to $f(b)=b^{0.5}(1-b)^{2}, \mathrm{Nb}-\mathrm{Ti}$ with the elongated grain boundaries can be described by $f(b)=b(1-b)$ and finally $\mathrm{Nb}_{3} \mathrm{Al}$ and $\mathrm{Nb}_{3} \mathrm{Ga}$ (by rapid processing) with defects on the atomic scale have the best performance at very high magnetic field and therefore can be described by $f(b)=b^{2}(1-b)$ dependence, see Fig. 2. The difference between these three types of pinning mechanism is not in the crystallographic structure of the grains but more in their morphology and intragranular defects, see Fig. 3. For the ordered array of the precipitates in superconductor (as it is presented in Fig. 2b) where the spacing between precipitates or superconducting phases with lower $T_{c}$ is equal to multiples of the flux line lattice 
spacing, a maximum in the $J_{c}$ vs. $B$ curve appears. Critical current peaks in $J_{c}$ vs. $B$ curves which are caused by such matching effects are easily identifiable because the flux densities where they appear are independent of temperature.

Considering described above differences between positions of the maximum pining force for various Nb-based materials, the most important consequence of a possible shift of the position of the maximum of a volume pinning force density $P_{v}$ as a function of $B$ in for $\mathrm{Nb}_{3} \mathrm{Sn}$ is that such materials could be used at liquid He temperature and also at liquid hydrogen temperatures (14 K). Such conductors if fully optimised will present a very substantial performance improvement as a function of magnetic flux density and also at elevated cryogenic temperatures as presented in Fig. 1b, becoming a viable alternative to MTS conductors.

\section{Improvement of $\mathrm{Nb}_{3} \mathrm{Sn}$ pinning}

The main reaction heat treatment to form the A15 layer in $\mathrm{Nb}_{3} \mathrm{Sn}$ bronze multifilamentary conductors is commonly an isothermal reactive diffusion in the range of $650{ }^{\circ} \mathrm{C}$ to $750^{\circ} \mathrm{C}$. At the lower end of this range the compound growth rate is very slow leading to unacceptably long reaction times [8], in addition the compound is disordered and has a high level of point defects exhibiting a degraded value of $T_{c}$ and $B_{c 2}[9]$. Above $750^{\circ} \mathrm{C}$ rapid grain growth and grain coarsening occurs [10], this is most apparent in the outermost region of the reaction layer which is the first to form, Fig. 4a. However for modern bronze processed ITER type strands with $\mathrm{Nb}$ filaments having diameter in the range of $2-3 \mu \mathrm{m}$ and subjected to the standard ITER heat treatment $\left(575^{\circ} \mathrm{C}\right.$ for $150 \mathrm{~h}+650^{\circ} \mathrm{C}$ for $200 \mathrm{~h}$ ) $\mathrm{Nb}_{3}$ Sn grains were mainly equiaxed but the grain size usually varied in relatively large interval from $30 \mathrm{~nm}$ to $250 \mathrm{~nm}$, Fig. 4b,c. It contradicts to earlier accepted scheme of 3-layered structure of $\mathrm{Nb}_{3} \mathrm{Sn}$ filaments that included two layers of equiaxed grains with different sizes and one layer of the columnar grains, Fig. 4a [11]. The results obtained are in good correlation with the data of recent scanning electron microscopy (SEM) investigation on $\mathrm{Nb}_{3} \mathrm{Sn}$ grains morphology in other ITER type strands [12].

Research conducted by Tarantini et al. [2] on the powder-in tube, $\mathrm{PIT}, \mathrm{Nb}_{3} \mathrm{Sn}$ conductors, where mixture of $\mathrm{NbSn}_{2}$, $\mathrm{Sn}$ and $\mathrm{Cu}$ powders is inserted in $\mathrm{Cu}$-clad $\mathrm{Nb}-$ 7.5 wt\% Ta tube and drawn in $\mathrm{Cu}$ matrix, address the fine grain structure layer formation of A15 phase influencing $J_{c}$ value [2]. The formation reaction of the fine grain structured $\mathrm{Nb}_{3} \mathrm{Sn}$ layer in such wires takes place as a reactive diffusion process between $\mathrm{NbSn}_{2}$ and $\mathrm{Sn}$ in presence of the $\mathrm{Cu}$ acting as a catalyst of fine grain formation as described previously in literature [13]. Such conductors are characterised by high density of grain boundaries in the small grain A15 layer, and such PIT conductor has an exceptionally high $J_{c}$ in the layer and also high specific grain boundary pinning force [2]. However, a substantial improvement of $J_{c}(B)$ was well documented, but there

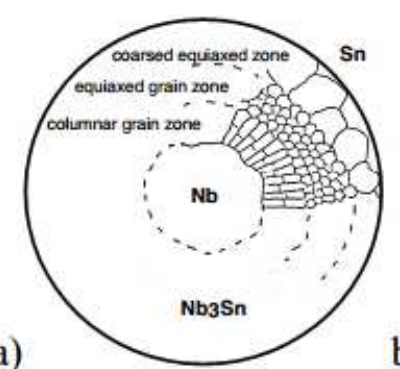

b)

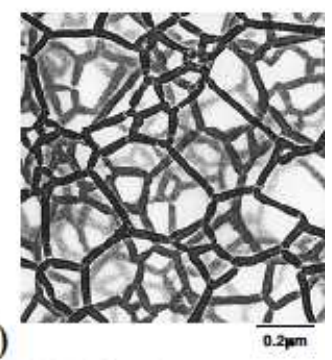

c)

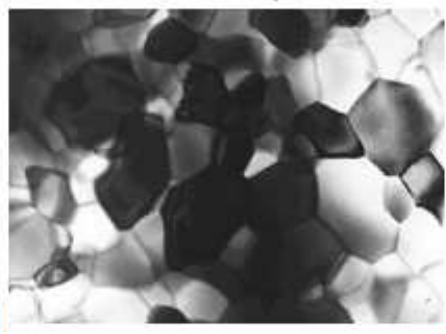

Fig. 4. (a) Cross-section of a single $\mathrm{Nb}-\mathrm{Nb}_{3} \mathrm{Sn}$ filament in the bronze matrix after solid state diffusion of $\mathrm{Sn}$ to $\mathrm{Nb}$ from the bronze matrix [14]. (b) Schematic projection of four $2 \mathrm{D}$ cuts through the $\mathrm{Nb}_{3} \mathrm{Sn}$ layers of a grain boundaries of the fine equiaxed zone [15]. $\mathrm{Nb}_{3} \mathrm{Sn}$ layer growth direction, normal to the picture. (c) TEM picture of the typical equiaxed zone; courtesy of $\mathrm{V}$. Pantsyrnyi.
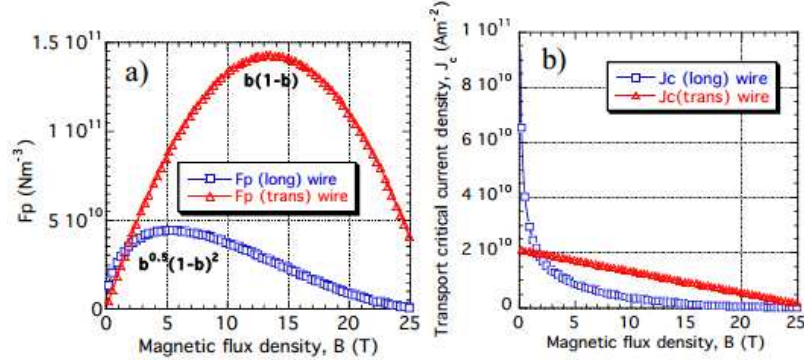

Fig. 5. (a) Pinning force versus magnetic flux density for both types of pinning: "transverse" pinning (elongated grains along the wire length) and "longitudinal" pinning (typical radial equiaxed grains) see Fig. 3 and 4; (b) critical current density for longitudinal and transverse pinning.

is no change in a pinning mechanism of the $\mathrm{Nb}_{3} \mathrm{Sn}$ layer which will shift the maximum pinning force position towards the higher magnetic flux density normalised values $B / B_{c 2}$. Therefore applicability of such conductors for liquid hydrogen cooled devices and high magnetic field applications is questionable.

As it was pointed out by Dew-Huges [16], Gurevich and Cooley [17] and later by McDonald and Barzi [18] the best solution to achieve higher pinning force at higher magnetic field would be an introduction of the pinning walls extended along the filaments direction by an analogy to the $\mathrm{Nb}-\mathrm{Ti}$ wires. The $\mathrm{Nb}_{3} \mathrm{Sn}$ grain boundary network provide continuous path for the flux lines, therefore flux can move entirely within the grain boundary regions and the pinning force can be defined by the shear between grain boundary flux and the stationary flux line 
lattice. Resulting field dependence of the critical Lorentz force for the most of the $\mathrm{Nb}_{3} \mathrm{Sn}$ conductors can be described as $b^{0.5}(1-b)^{2}$. In $\mathrm{Nb}-\mathrm{Ti}$ the grain boundaries, drawn out parallel to filament axis act as effective barriers to flux motion. Obviously there is also a contribution from $\alpha$-Ti precipitation at the grain boundaries, dislocation tangles, sub-grain boundaries and interfaces with non-superconducting a-Ti precipitation but the overall morphology of the elongated grains has its strong influence which results in critical Lorentz force vs. field dependence for the most of the Nb-Ti close to $b(1-b)$ (Eq. (2), Fig. 3a). For effective pinning the morphology of the noodle-shaped particles perpendicular to the wire axis is very important. Only those pins having their flat area perpendicular to the Lorentz force direction can be active [19].

Based on the earlier calculations of [16-18] the "transverse" pinning, corresponding mainly to NbTi can be expressed as Eq. (3):

$$
J_{c, \text { trans }}(B, T)=\frac{B_{c 2}}{8 \pi \mu_{0} \kappa_{1}^{2} D}\left(1-\frac{B}{B_{c 2}}\right) .
$$

But "longitudinal" pinning mainly describing $\mathrm{Nb}_{3} \mathrm{Sn}$ conductors can be expressed as Eq. (4):

$$
J_{c, \text { long }}(B, T)=\frac{2 \alpha d B_{c 2}^{2}}{3 \sqrt{3 \Phi_{0}} \mu_{0} \kappa_{1}^{2} D} \frac{\left\langle J_{0}\right\rangle^{2}}{J_{d}} \frac{\left(1-\frac{B}{B_{c 2}}\right)^{2}}{\sqrt{B}} .
$$

Parameter $d$ is an effective size of the pinning site proportional to the effective boundary thickness, $J_{d}$ is the depairing current density of the grains, $a=\delta J_{0} /\left\langle J_{0}\right\rangle$ is the ratio of the decrement in $J_{0}, \delta J_{0}$ to the average value, $\left\langle J_{0}\right\rangle, D$ is a grain size, $1 / D$ is the density of pinning sites, $k_{1}=B_{c 2} / 2^{0.5} B_{c}, B_{c}(T)=B_{c}(0)\left[1-\left(T / T_{c 0}\right)^{2}\right]$.

Results of such calculations addressed to the possible conductors with the longitudinal and transversal pinning taking to account the actual material parameters: $\left\langle J_{0}\right\rangle / J_{d}=0.7, B_{c 0}=27 \mathrm{~T}, T_{c 0}=19 \mathrm{~K}, T=4.2 \mathrm{~K}$, $a=0.25, d=2 \mathrm{~nm}, k_{1}=20$ and $D=100 \mathrm{~nm}$ are presented in Fig. 5.

Results presented in Fig. 5 are very encouraging, suggesting that a substantial improvement of the future $\mathrm{Nb}_{3} \mathrm{Sn}$ conductors could be possible if only with extended ribbon-like defects along the conductor axis would be produced. To study the influence of $\mathrm{Nb}_{3} \mathrm{Sn}$ grain morphology on the pinning in the case of round multifilamentary conductors is rather difficult due to the round fibre symmetry of the filaments. There have been attempts to make a general calculation of the average $\mathrm{Nb}_{3} \mathrm{Sn}$ grain size based on the computation of the large number of the experimental data [20]. Although such regression analysis with independent processing parameters such as diffusion time, diffusion temperature, number of niobium filaments, diameter of $\mathrm{Nb}$ filaments and volume ratio of the $\mathrm{Cu}-\mathrm{Sn}$ matrix to $\mathrm{Nb}$ filaments provides correct results but only valid for the equiaxed-grain layers formed in a bronze wires, see Fig. 4.

The grain morphology and stoichiometry of the $\mathrm{Nb}_{3} \mathrm{Sn}$ superconducting layers formed at different temperatures during liquid reactive diffusion (LRD) processes in long length conductors presented in Fig. 6 differ noticeably and may provide additional information on the possible improvement of the pinning force described by Eq. (3) and Eq. (4). The diffraction patterns of the investigated $\mathrm{Nb}_{3} \mathrm{Sn}$ layers were found not to differ from one another and they were practically the same as those in a randomly oriented $\mathrm{Nb}_{3} \mathrm{Sn}$ polycrystalline material.

For the lower temperatures and shorter times of the LRD process the grains are smaller and ellipsoidal in shape with the longer axis parallel to the surface, therefore $F_{p}(H \| S) \gg F_{p}(H \perp S)$, Fig. 6a and Fig. 7a and b whereas during diffusion at higher temperatures at also for longer time $\mathrm{Nb}_{3} \mathrm{Sn}$ grains become bigger and grow more perpendicularly to the surface of the $\mathrm{Nb}-1.5 \% \mathrm{Zr}$ substrate. In this case the value of the $F_{p}(H \| S) \cong$ $F_{p}(H \perp S)$, Fig. $6 \mathrm{~b}$ and Fig. $7 \mathrm{a}$ and c. This may look like simple case but there is an evidence that in other A15 coated conductors there is crossover between $F_{p}(H \| S)$ and $F_{p}(H \perp S)$ versus external magnetic field. At higher magnetic fields it is possible that the mechanism may change and columnar grains may be more efficient for $F_{p}(B \| S)$. The volume pinning force anisotropy measurements conducted on these tapes in external magnetic fields proved that the differences induced by the grain morphology are noticeable (see Fig. 7a) and confirm need for further study of the grain morphology improvement for better conductor performance in magnetic field. a)

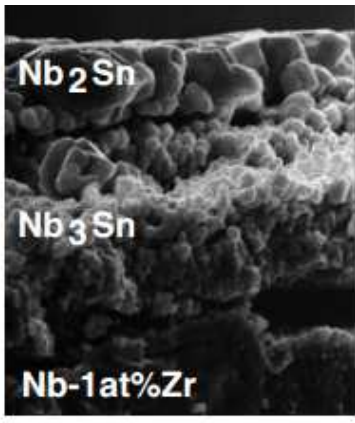

b)

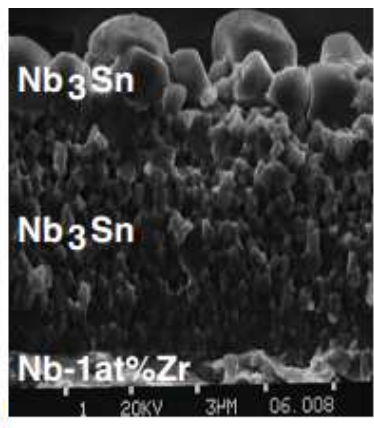

Fig. 6. Nb-Sn layers manufactured by liquid reactive diffusion process: (a) equiaxed grains and of the ellipsoidal shape with longer axis along the tape surface, $930^{\circ} \mathrm{C}, 11 \mathrm{~min}$; (b) oval columnar grains partially perpendicular to the surface, $980^{\circ} \mathrm{C}, 11 \mathrm{~min}$ [13].

Important pinning measurements results conducted for $\mathrm{Nb}_{3} \mathrm{Ge}$ coated conductor (Fig. 8a) suggest that possibly two different types of pinning mechanisms are measured for the same superconducting layer, depending on the magnetic field orientation to the film surface [22]. The $\mathrm{Nb}_{3} \mathrm{Ge}$ layer was of columnar structure so the effective density of flux-pinning centres in the directions parallel and perpendicular to the growth direction of the columnar grains are very different. It can be assumed that "point-like" forces (pinning forces induced by the point defects) would be expected to dominate in the field perpendicular to the columnar grain axis, while "line-like" 

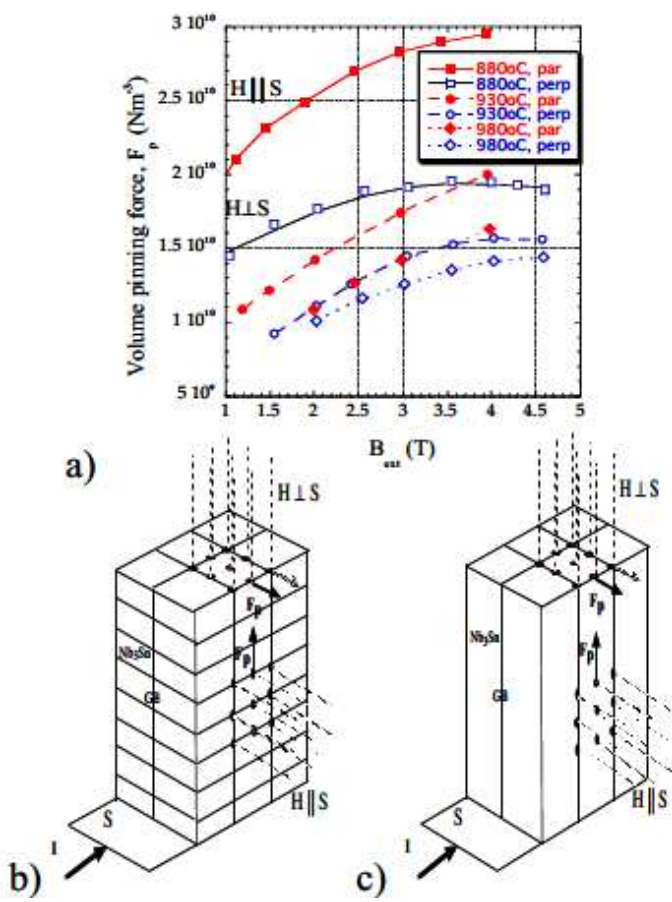

Fig. 7. (a) Dependence of volume pinning force $F_{p}$ vs. $B_{\text {ext }}$ for $\mathrm{Nb}_{3} \mathrm{Sn}$ coated conductor manufactured by LRD process, of tin atoms from the Cu80at\%Sn to $20 \mathrm{~mm}$ thick, $1 \mathrm{~cm}$ wide $\mathrm{Nb}-1.5 \% \mathrm{Zr}$ tapes for $11 \mathrm{~min}$ in vacuum [21]. (b) Schematic representation of the grain boundary pinning for the $\mathrm{Nb}_{3} \mathrm{Sn}$ flat-like grains (see Fig. 6a). (c) Schematic representation of the grain boundary pinning for the $\mathrm{Nb}_{3} \mathrm{Sn}$ columnar grains (see Fig. 6b). For simplification the square-like flux lattice was considered. It is evident that if the GB pinning dominates the higher pinning force should be reached for $H \perp S$. Magnetic field was always perpendicular to the current flow direction.

or "area-like" pinning forces may be caused by the grain boundary surfaces in the parallel to the film surface field. On the other hand, at higher magnetic field where collective pinning on the intragrain defects dominates the effectiveness of the interconnected grain boundary, pinning will be reduced and the higher pinning force should be reached for $H \| S$.

Despite the substantial differences in $F_{p}$ observed for the tape conductor, result of pinning force summation (Fig. 8b) showed a limited improvement of the overall performance of the wires because the calculated $F_{p} / F_{p \max }(b)$ dependence is very similar to $b^{0.5}(1-b)^{2}$. Successful simulation of the $F_{p}$ vs. $B$ dependences in Fig. 8 using Eq. (3) and Eq. (4) can be conducted assuming that only parameter to be changed in Eq. (4) is an effective grain boundary size parameter $d$. By increasing $d$ from $2 \mathrm{~nm}$ to $10 \mathrm{~nm}$ in Eq. (4) the results presented in Fig. 9 are identical to those in Fig. 8a.

Despite the substantial differences in $F_{p}$ observed for the tape conductor, result of pinning force summation (Fig. 8b) showed a limited improvement of the overall performance of the wires because the calculated $F_{p} / F_{p \max }(b)$ dependence is very similar to $b^{0.5}(1-b)^{2}$.
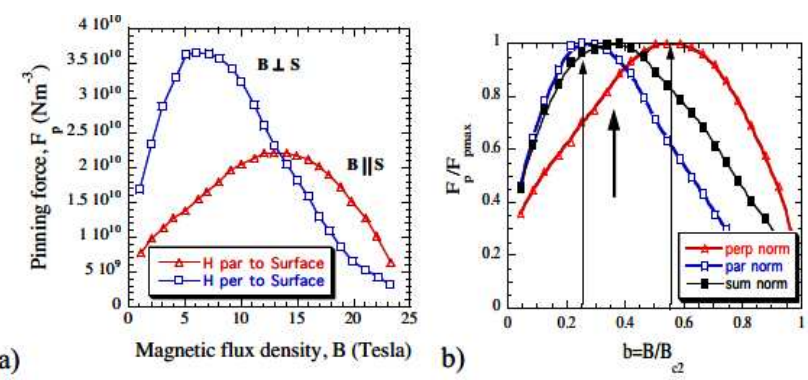

Fig. 8. (a) Global pinning-force density of tape $\mathrm{Nb}_{3} \mathrm{Ge}$ coated conductor manufactured by CVD [22]; $H \perp S$ represents magnetic field perpendicular to the tape surface but parallel to the columnar grains longer axis, $H \| S$ represents magnetic field parallel to the tape surface, but perpendicular to the columnar longer axis. After Suzuki et al. [23]. (b) Normalised pinning force for the data from Fig. 8a. An additional curve in Fig. 8b represents the expected resulting performance of the tape conductor which is very similar to $b^{0.5}(1-b)^{2}$, Fig. 3 .
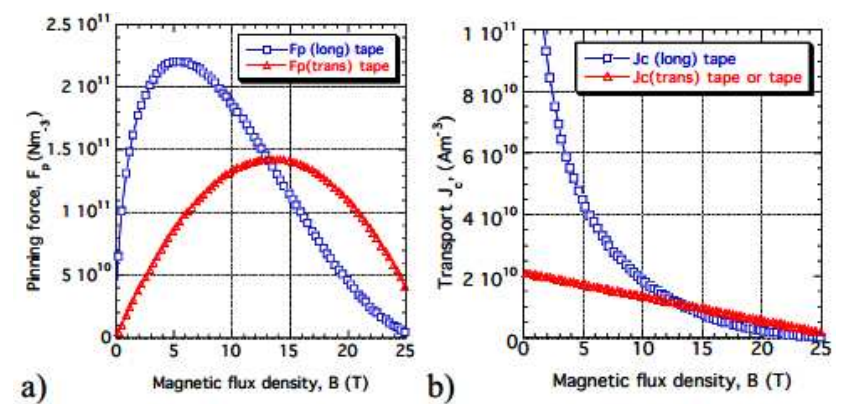

Fig. 9. (a) Pinning force versus magnetic flux density for both types of pinning: "transverse" pinning (elongated grains along the wire length) and "longitudinal" pinning (typical radial equiaxed grains), see Fig. 6 . (b) Critical current density for longitudinal and transverse pinning.

Successful simulation of the $F_{p}$ vs. $B$ dependences in Fig. 8 using Eq. (3) and Eq. (4) can be conducted assuming that only parameter to be changed in Eq. (4) is an effective grain boundary size parameter $d$. By increasing $d$ from $2 \mathrm{~nm}$ to $10 \mathrm{~nm}$ in Eq. (4) the results presented in Fig. 9 are identical to those in Fig. 8a.

In this case A15 columnar grains for magnetic field perpendicular to substrates can be described by Eq. (4) as a longitudinal pinning (along most of the grain boundaries) whereas for magnetic field parallel to substrates it can be described by Eq. (3) as a transversal pinning (along smaller number of the grain boundaries).

Despite of clear evidence that Eq. (3) and Eq. (4) well describe a wide range of $\mathrm{Nb}_{3} \mathrm{Sn}$ morphologies, more dramatic changes in the grain boundary structure are required if the $\mathrm{Nb}_{3} \mathrm{Sn}$ wires are going to achieve the improved field performance, $f(b)=b(1-b)$ and also $J_{c}$ vs. $B$. There are indications in the literature that controlled texture development of the Nb-based sub- 
strates/filaments may influence grain morphology of $\mathrm{Nb}_{3} \mathrm{Sn}$ layer. Microstructural differences of $\mathrm{Nb}_{3} \mathrm{Sn}$ superconducting diffusion layers grown by tin reaction with single niobium crystal substrate with faces parallel to the major bcc planes (110),(100), (211) and (111) have been presented in literature $[24,25]$. The results showed that $\mathrm{Nb}_{3} \mathrm{Sn}$ layers grown on the surfaces (211), (100) and (110) of $\mathrm{Nb}$ substrate have a similar morphology, minor variations in size and grain coverage, while layers grown on the $\mathrm{Nb}$ (111) surface have grains which are not only jagged but also highly irregular in size and distribution. The same grain morphology was observed for $\mathrm{Nb}_{3} \mathrm{Sn}$ layers grown on a polycrystalline $\mathrm{Nb}$ substrate of (111) main texture [26].

The microscopical investigations revealed a welldefined planar fiber structure produced by the cold rolling. Transport critical current density was found lower when fibers are parallel to the direction of magnetic field than when they are perpendicular. This effect may be a consequence of the interaction of flux movement with fiber boundaries [27]. Research on the effect of the pre-reaction of the bronze conductors on the properties of the $\mathrm{Nb}_{3} \mathrm{Sn}$ composites revealed significant circumferential differences in the grain morphology of the fine filaments which may be due to the crystallographic orientations developed during cold rolling of Nb filaments [28].

There is a clear evidence in the literature which might suggest that texture induced growth of $\mathrm{Nb}_{3} \mathrm{Sn}$ may play a role in further improvement of pinning. Pole figures indicate that the main feature of the texture in the $\mathrm{Nb}_{3} \mathrm{Sn}$ layers is identical with that in the niobium substrate irrespectively of the processing technique [5, 29].

\section{Conclusions}

Presented discussion and presented methods of possible methods of improvement of pinning in the grain boundary dominating pinning mechanism of $\mathrm{Nb}_{3} \mathrm{Sn}$ wires highlights a potential solution for the wires to operate at the higher magnetic flux densities $\approx 10-15 \mathrm{~T}$ range in a slash indirect hydrogen cooling system $(14 \mathrm{~K})$ where circulating compress helium gas is a force cooling medium as in detail was presented.

This important findings should be further researched experimentally to establish practical limits on such very needed enhancement of practical $\mathrm{Nb}_{3} \mathrm{Sn}$ conductors.

\section{References}

[1] Z. Cai, R.H. Clarke, B.A. Glowacki, W.J. Nuttall, N. Ward, Resources Policy 35, 77 (2010).

[2] C. Tarantini, C. Segal, Z.H. Sung, P.J. Lee, L. Oberli, A. Ballarino, L. Bottura, D.C. Larbalestier, Supercond. Sci. Technol. 28, 095001 (2015).
[3] B.A. Glowacki, M. Majoros, M. Vicker, M. Eisterer, S. Toenies, H.W. Weber, M. Fukutomi, K. Komori, K. Togano, Supercond. Sci. Technol. 16, 297 (2003).

[4] L.T. Summers, J.R. Miller, IEEE Trans. Magn. 23, 1552 (1987).

[5] B.A. Glowacki, Intermetallics 7, 117 (1999).

[6] E.J. Kramer, J. Appl. Phys. 44, 1360 (1973).

[7] H. Ullmaier, Irreversible Properties of Type II Superconductors, Springer-Verlag, Berlin 1975.

[8] S. Ochiai, K. Osamura, M. Ryoji, Acta Metall. 35, 1433 (1987).

[9] W. Schaure, W. Schelb, IEEE Trans. Magn. 17, 374 (1981).

[10] W. Schelb, J. Mater. Sci. 16, 2575 (1981).

[11] W. Wu, D.R. Dietderich, J.T. Holthuis, M. Hong, W.V. Hassenzahl, J.W. Morris, J. Appl. Phys. 54, 7139 (1983).

[12] P.J. Lee, C.M. Fisher, M.T. Naus, A.A. Squitieri, D.C. Larbalestier, IEEE Trans. Appl. Supercond. 13, 3422 (2003)

[13] B.A. Glowacki, J. Mater. Sci. Lett. 4, 389 (1985).

[14] N.J. Pugh, J.L.M. Robertson, E.R. Wallach, J.R. Cave, R.E. Somekh, J.E. Evetts, IEEE Trans. Magn. 21, 1129 (1985).

[15] B.A. Glowacki, Ph.D. Thesis, IBSiNT, Polish Academy of Sciences, Wrocław, Poland 1983.

[16] D. Dew-Huges, IEEE Trans. Magn. 23, 1172 (1987).

[17] A. Gurevich, L.D. Cooley, Phys. Rev. B 50, 13563 (1994).

[18] J. McDonald, E. Barzi, IEEE Trans. Appl. Supercond. 11, 3884 (2001).

[19] H. Hillman, Supercond. Sci. Technol. 12, 348 (1999).

[20] X. Aiying, H. Qingyong, S. Fengming, F. Juren, Cryogenics 25, 507 (1985).

[21] B.A. Glowacki, M. Horobiowski, Phys. Status Solidi A 89, K13 (1985).

[22] J.J. Hanak, H.S. Berman, J. Phys. Chem. Solids C7, 249 (1967), Supplement on Proc. Intern. Conf. Crystal Growth.

[23] M. Suzuki, Y. Watanabe, T. Anayama, K. Watanabe, K. Noto, Jpn. J. Appl. Phys. 26, 881 (1987).

[24] V. Diaduk, J. Bostock, M.L.A. MacVicar, IEEE Trans. Magn. 15, 610 (1979).

[25] B. Hillenbrand, Y. Uzel, K. Schmitzke, Appl. Phys. 23, 237 (1980).

[26] B.A. Glowacki, Phys. Status Solidi A 75, K103 (1983).

[27] D. Kramer, C.G. Rhodes, Trans. Metall. Soc. AIME 233, 192 (1965).

[28] D.B. Smathers, K.R. Marken, D.C. Larbalestier R.M. Scanlan, IEEE Trans. Magn. 19, 1417 (1983).

[29] K. Togano, K. Tachikawa, J. Appl. Phys. 50, 3495 (1979). 